\title{
Widening the spectrum of filamin-C myopathy: Predominantly proximal myopathy due to the p.A193T mutation in the actin-binding domain of FLNC
}

\author{
Fleur J.A. van den Bogaart a, Kristl G. Claeys ${ }^{\text {b,c }}$, Rudolf A. Kley ${ }^{\mathrm{d}}$, Benno Kusters ${ }^{\mathrm{e}}$, \\ Simone Schrading ${ }^{\text {f }}$, Erik J. Kamsteeg ${ }^{g}$, Nicol C. Voermans ${ }^{a}{ }^{a} *$ \\ a Department of Neurology, Radboud University Medical Centre, Nijmegen, The Netherlands \\ ${ }^{\mathrm{b}}$ Department of Neurology and Institute of Neuropathology, RWTH Aachen University, Aachen, Germany \\ ' Department of Neurology, University Hospitals Leuven and University of Leuven (KU Leuven), Leuven, Belgium \\ ${ }^{\mathrm{d}}$ Department of Neurology, Heimer Institute for Muscle Research, Ruhr-University Bochum, Bochum, Germany \\ ${ }^{\mathrm{e}}$ Department of Pathology, Radboud University Medical Centre, Nijmegen, The Netherlands \\ ${ }_{\mathrm{f}}^{\mathrm{f}}$ Department of Radiology, RWTH Aachen University, Aachen, Germany \\ ${ }^{g}$ Department of Human Genetics, Radboud University Medical Centre, The Netherlands
}

Received 10 April 2016; received in revised form 13 September 2016; accepted 20 September 2016

\begin{abstract}
We report three patients with a predominantly proximal myopathy due to p.A193T mutation in the actin-binding domain of $F L N C$, which has so far only been associated with a distal myopathy. They presented with a late onset myopathy characterized by predominant limb-girdle and proximal weakness. We describe the clinical, electrophysiological, pathological, muscle imaging and genetic features. One of our patients did not have typical histological features for a myofibrillar myopathy in muscle biopsy. This observation is important for the recognition of the full clinical spectrum of filamin-C-related myopathies. Muscle imaging has an important role in distinguishing the different filamin-C myopathy types.

(C) 2016 Published by Elsevier B.V.
\end{abstract}

Keywords: Myopathy; Filamin-C; FLNC mutation

\section{Introduction}

Filamin-C-related myopathies are autosomal dominant inherited myopathies caused by mutations in $F L N C$, the gene encoding filamin-C. Three distinct types of myopathies have been described [1-4]. First, pathogenic mutations in the rod domain of FLNC generally cause a myofibrillar myopathy (MFM), histologically defined and characterized by focal disintegration of myofibrils and by formation of large sarcoplasmic protein aggregates [5]. MFM-filamin-C myopathy is usually associated with slowly progressive weakness predominantly affecting proximal muscles and starting in the fourth-to-sixth decade of life $[1,6,7]$. The two other filamin-C myopathy types have been described as distal myopathies with histological features not fulfilling the criteria required for

\footnotetext{
* Corresponding author. Neuromuscular Centre Nijmegen, Department of Neurology, 935, Radboud University Medical Centre, P.O. Box 9101, 6500 HB Nijmegen, The Netherlands. Fax: +31243541122 .

E-mail address: nicol.voermans@radboudumc.nl (N.C. Voermans).
}

diagnosis of MFM. One is caused by a frameshift mutation in FLNC that leads to a haploinsufficiency [2] and the other by missense mutations in the actin-binding domain of filamin-C resulting in increased actin binding affinity [3].

We here describe three patients from two non-related families with a p.A193T mutation in the actin-binding domain of FLNC, all presenting with a predominantly proximal myopathy but with a more variable phenotype than the MFM subtype. This report thus adds to the clinical spectrum of filamin-C-related myopathies and can aid in clinical recognition of this rare disorder.

\section{Case report}

\subsection{Patient 1}

A 53-year-old woman presented with mildly elevated serum creatine kinase levels (301 U/l) and gradually progressive limb girdle weakness since her forties. Initially, this was considered to be secondary to hypothyroidism and statin use. However, supplementation of thyroid hormone and cessation of statin therapy had not reduced her symptoms. Her walking distance 

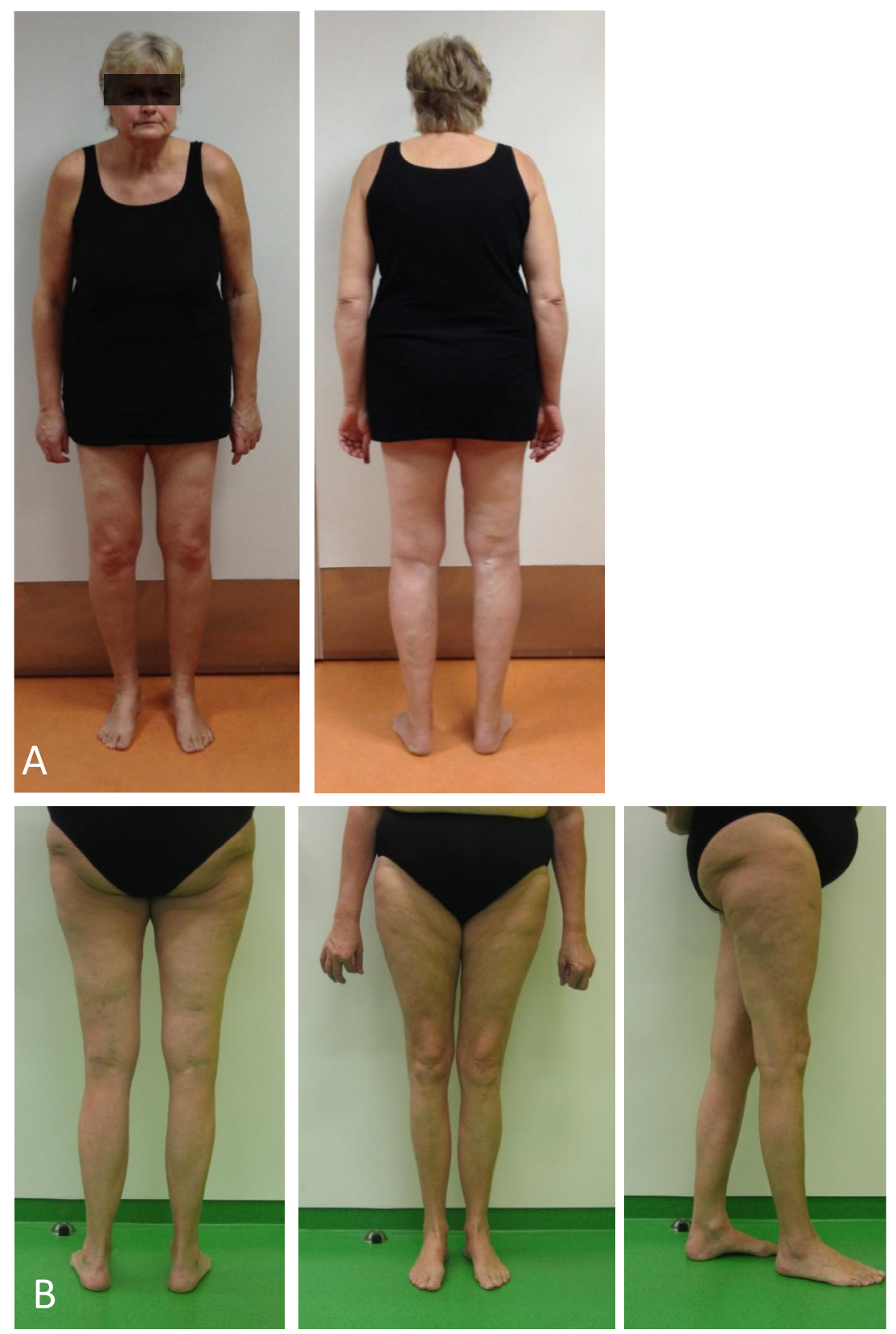

Fig. 1. Proximal and distal muscle atrophy in patient 1 (A) and patient 3 (B).

was limited to 500 metres, she could not run anymore and had difficulties climbing stairs. She experienced a feeling of weakness at the knees, without experiencing falls. She had kyphoscoliosis since childhood and had never been good at sports. Her medical history included a short episode of cardiac arrhythmia which was successfully treated with ACE inhibitors. Her mother, grandmother and great aunt also had slowly progressive muscle weakness, but without a specific diagnosis.

Physical examination revealed muscle weakness in truncal muscles, infraspinatus and supraspinatus muscles (MRC 4/5), neck flexion [4], hip flexion [4] and hip extension [2]. Distal strength in her arms was normal. Gowers' sign was present. The gait was waddling and walking on toes and heels was not possible. Atrophy was present in distal legs and quadriceps muscles bilaterally (Fig. 1A). Deep tendon reflexes (DTR) were absent. Vibration, pain and temperature sense were reduced in both legs distally.

Electromyography (EMG; at age 48 years) of the anterior tibial, rectus femoris, biceps brachii, and deltoid muscles showed no myopathic changes (no small or polyphasic motor unit action potentials (MUAP)). Muscle biopsy (at age 48 years) from the lateral vastus muscle showed minor abnormalities: few hypertrophic and some atrophic fibres, and focal changes that could have been suggestive for fibre type grouping (Fig. 2A).

Oxidative staining showed some inhomogeneous pattern, occasionally with some core-like appearance (Fig. 2B), but electron microscopy (EM) did not exhibit a significant core 
A

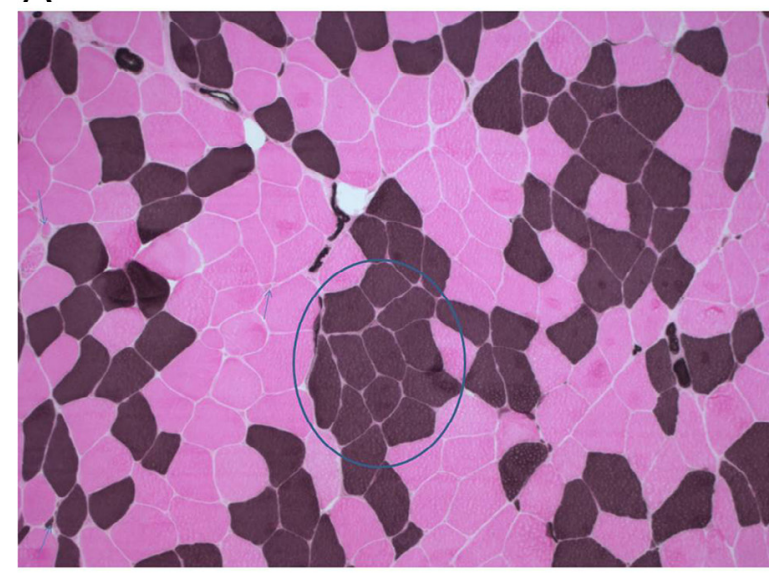

B

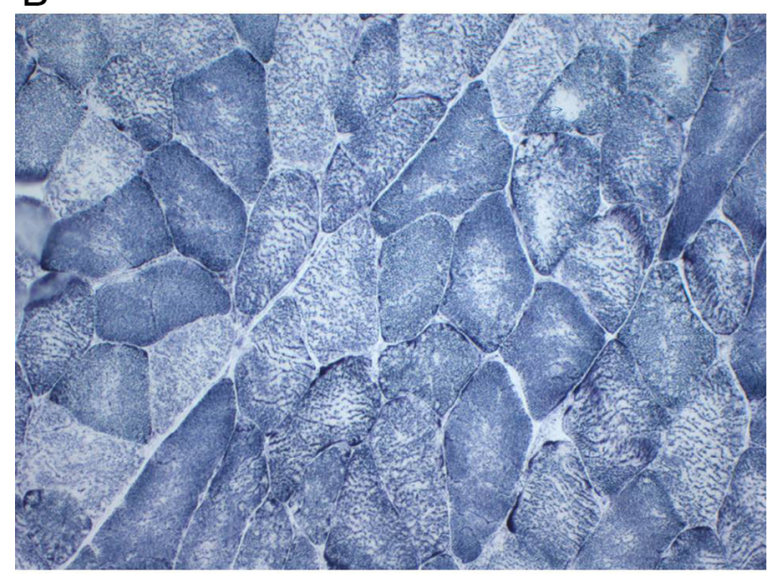

C

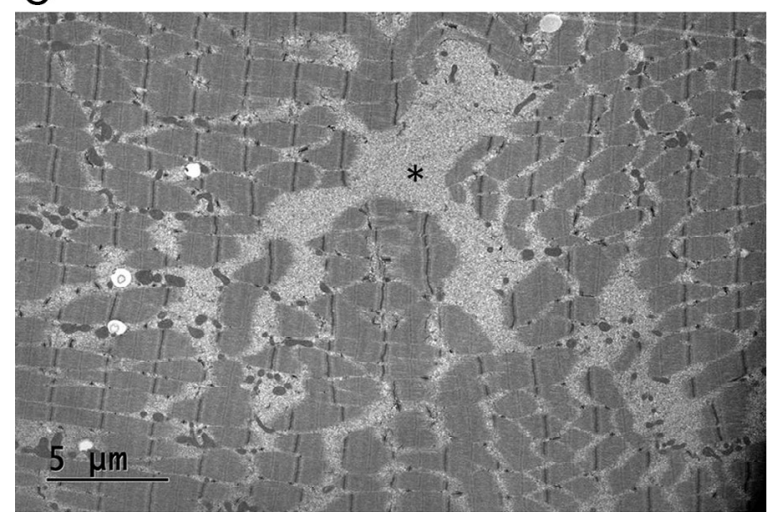

Fig. 2. Muscle biopsy of patient 1. (A) ATPase ( $\mathrm{pH}$ 4.2) staining showing occasionally atrophic fibres (arrows) and focal fibre type clustering, but fibre type grouping (circled) was not definite. (B) SDH-staining showing some inhomogeneous distribution pattern, sometimes resembling core-like structures (arrow). (C) Electron microscopy with the presence of glycogen (*), an uneven distribution of mitochondria and the absence of myofibrils.

pathology (Fig. 2C). Western blot of the muscle was normal for calpain-3, dysferlin and dystrophin. Cardiac screening (ultrasound and $24 \mathrm{~h}$-Holter-ECG) showed no abnormalities. Muscle MRI of lower limbs at the age of 50 years showed fatty degeneration of several muscles. At the level of the thighs, the vastus medialis, vastus intermedius, vastus lateralis, semimembranosus and the long head of the biceps femoris muscles were affected. The gracilis, sartorius, rectus femoris, semitendinosus and adductor muscles were relatively spared. At the lower leg level, the soleus, gastrocnemius medialis, gastrocnemius lateralis and peroneal group muscles were involved (Fig. 3A). Nerve conduction studies (NCS; ulnar, posterior tibial, and common peroneal (motor); radial and sural nerve (sensory)) at the age of 53 years were normal. A repeated EMG at the age of 53 years showed mildly myopathic changes in the gastrocnemius muscle (small, polyphasic MUAP). Repeated CK was 177 U/1. Proximal myotonic myopathy was genetically excluded, and no mutations in COL6A1/2/3 (tested because of the kyphoscoliosis) or RYRI were detected by sequencing. Whole exome sequencing (age 53 years) identified a heterozygous c.577G $>$ A, p.A193T mutation in the actinbinding domain of FLNC.

\subsection{Patient 2}

The mother of patient 1 was investigated at the age of 79 years. She had been wearing a corset for scoliosis since the age of 15 years. Throughout adulthood, she gradually developed proximal muscle weakness. At the age of 53 years, she was assessed by a neurologist, who had reported mild muscle weakness of hip and shoulder girdle. She used a cane or rollator to walk and became wheelchair dependent in her 60s. Respiratory weakness with hypoventilation became apparent at the age of 75 years. Her medical history revealed diabetes, subclinical hypothyroidism, arterial hypertension and glaucoma.

At age 79 years, muscle weakness was still more pronounced proximally: shoulder abduction (MRC 2/5), interosseous muscles [3], finger flexors and extensors [4], iliopsoas muscle [3], quadriceps muscle [4], gluteal muscles [2] and biceps femoris [3]. DTR and vibration sense in the legs were reduced. She died at the age of 82 years due to a respiratory complication after a leg fracture. The heterozygous c.577G $>$ A, p.A193T mutation in FLNC, was detected post-mortem.

\subsection{Patient 3}

This unrelated 58-year-old woman presented with progressive proximal muscle weakness since the age of 54 years. She stumbled frequently, without falling. The patient had difficulties walking fast, climbing stairs and rising from a squatting position or chair. She had exertional dyspnoea and could not ride a bike for more than 30 minutes. Her parents, brother and 14-year-old daughter were asymptomatic.

Physical examination showed a waddling gait, and walking on toes or heels was not possible. Gowers' sign was present. Atrophy of the muscles was seen in distal legs and quadriceps muscles bilaterally (Fig. 1B). Muscle strength in arms and hands was normal. Both proximal and distal symmetrical muscle weakness was observed in her legs: iliopsoas and quadriceps muscle (MRC 3/5); biceps femoris, semitendinosus and semimembranosus [3], tibialis anterior [2], gastrocnemius muscle [2], leg adductors and abductors [3]. Temperature sense was reduced in both feet and vibration sense was reduced to $2 / 8$ on the left and 0/8 on the right malleolus. DTR were decreased in the upper limbs and absent in the legs. 
A
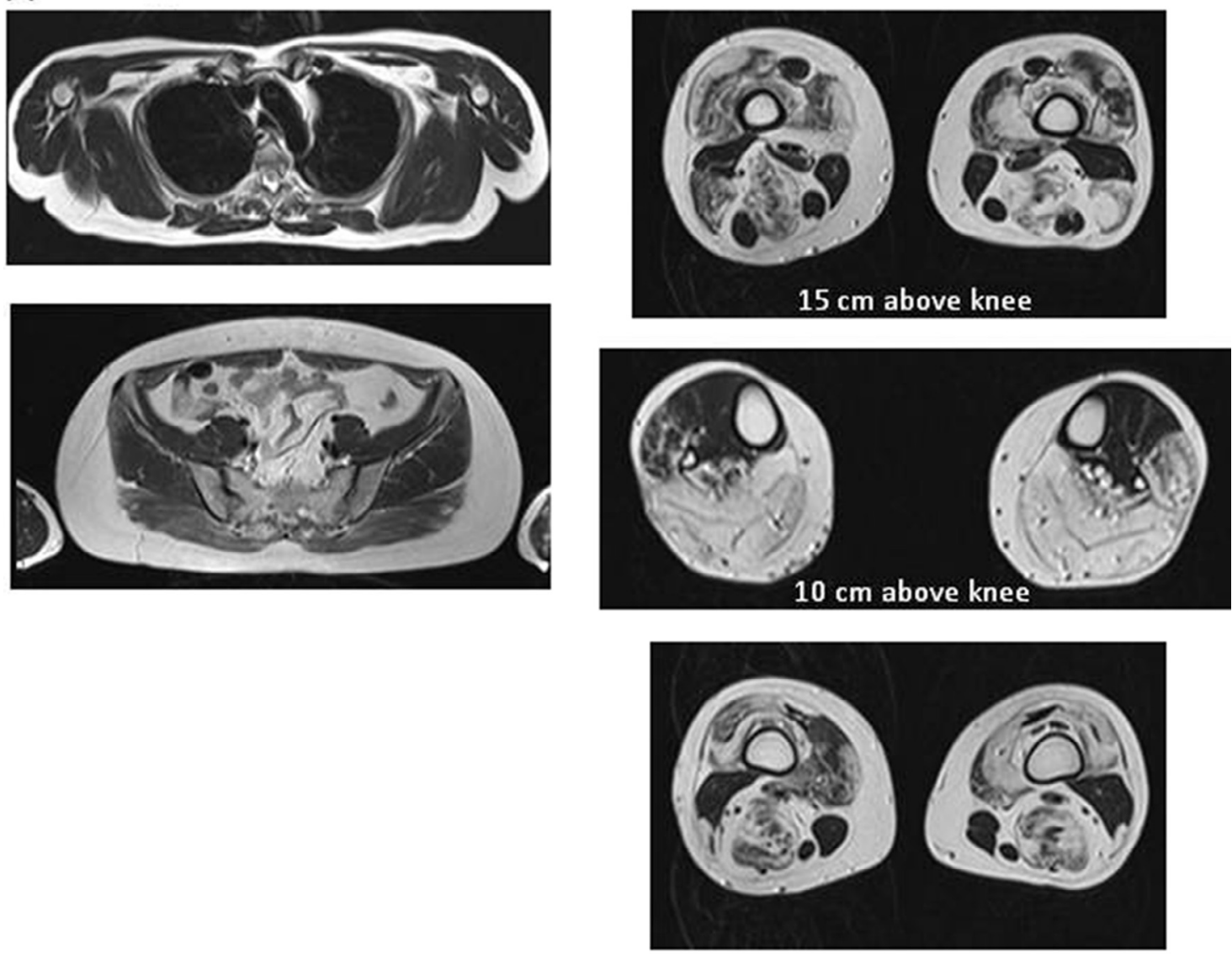

B
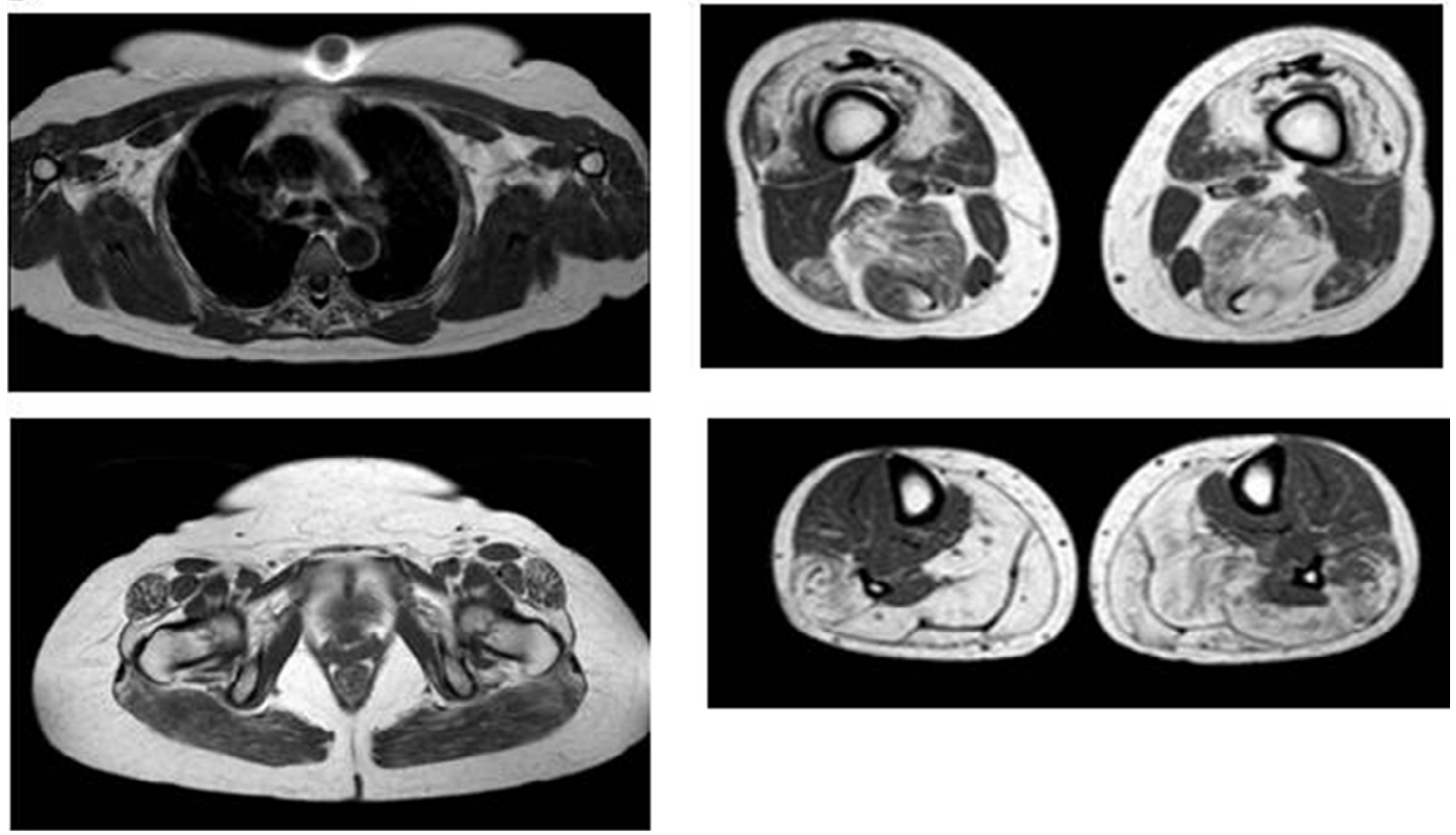

Fig. 3. Transverse T1-weighted muscle MRI in patient 1 and patient 3. (A) (patient 1): Shoulder and upper arms: no fatty infiltration or atrophy. Hip girdle: mild fatty infiltration of the gluteus maximus muscle bilaterally. Upper leg: lipomatous alterations of the vastus medialis, intermedius and lateralis, the long head of the biceps femoris and the semimembranosus. Lower leg: fatty degeneration of the soleus, gastrocnemius medialis, gastrocnemius lateralis and flexor halluces longus muscles and to a lesser extent of the peroneal group muscles. (B) (patient 3): Shoulder and upper arms: no fatty infiltration or atrophy as far as this could be assessed. Hip girdle: moderate lipomatous alterations of tensor fascia latae and the iliacus muscles. Upper leg: fatty degeneration of the vastus medialis, intermedius and lateralis, biceps femoris, semitendinosus and semimembranosus. The adductor magnus is only slightly affected. Lower leg: severe involvement of the gastrocnemius and soleus muscles. 
Cardiac screening was normal (24 h-Holter-ECG and echocardiography). Vital capacity was $89 \%$ and CK-levels were normal (106 U/l). EMG showed no myopathic changes in the anterior tibial, gastrocnemius, and bilateral medial vastus muscles, and NCS (tibial nerve bilaterally (motor); sural nerve bilaterally) were normal. Muscle MRI showed a similar pattern of lower limb muscle involvement as observed in patient 1, but the semitendinosus muscle was more affected (Fig. 3B). Pompe disease and limb-girdle muscular dystrophy type $2 \mathrm{~L}$ (LGMD2L) were excluded by an assessment of enzymatic activity and Sanger sequencing of the ANO5-gene.

Next-generation sequencing (NGS) panel genetic analysis revealed the heterozygous c. $577 \mathrm{G}>\mathrm{A}$, p.A193T mutation in FLNC.

\section{Discussion}

We here present three patients from two unrelated families with the p.A193T mutation in the actin-binding domain of FLNC associated with a phenotype of predominant limb-girdle and proximal weakness. The previously reported distal phenotype associated with mutations in this domain of filamin-C [3] apparently only represents part of the full filamin-C myopathy spectrum.

In 2011, two different mutations in the actin-binding domain of FLNC, p.A193T and p.M251T, have been reported in a family from Australia and Italy, respectively. Affected members of these families presented with a distal myopathy starting in the third decade of life in the hands with a characteristic thenar muscle weakness, followed by posterior calf muscle involvement in the fourth and proximal weakness in the fifth decade of life [3]. The clinical phenotype of the three patients harbouring the p.A193T mutation in this report was clearly different. Weakness manifested above age 40 years in proximal lower limbs and involved distal and respiratory muscles later in the disease course similar as described in the MFM subtype caused by mutations in the rod domain of FLNC. However, histological changes in patient 1 were not specific for MFM. Furthermore, muscle imaging findings in lower legs of patient 3 were typical of filamin-C-related distal myopathy caused by mutations in the actin-binding domain of filamin-C [3], but also showed distinct fatty infiltration of thigh muscles. Compared to the MFM subtype, the vastus lateralis was more affected, while the adductor magnus, gastrocnemius lateralis and anterior lower leg muscles were less affected $[8,9]$.

These different and specific patterns of muscle involvement underline that muscle imaging is useful in the diagnosis of hereditary myopathies and to distinguish between different filamin-C related myopathy types. The absence of fatty replacement in the vastus lateralis sample of patient 1 may be explained by the fact that muscle biopsy was performed two years before muscle imaging in this patient.

Interestingly, the p.A193T mutation detected in the patients in this cohort is identical to the one reported in distal myopathy. The molecular mechanisms causing myopathy seem to include an increased actin binding of this filamin $\mathrm{C}$ mutant [3]. This suggests that both phenotypes, distal myopathy and proximal myopathy, are within the same disease spectrum rather than allelic disorders. The differences between the two may be due to yet unknown genetic and environmental causes.

In short, these data indicate that the phenotype of filaminC-related myopathy due to mutations in the actin binding domain is more variable than previously known. Hence, mutations in FLNC should be considered in patients with proximal myopathy and late disease onset even if histological features of MFM are lacking. This observation is important for scheduling genetic testing in myopathy patients.

\section{Acknowledgments}

We would like to acknowledge the Genome Technology Center at the Radboudumc and the Beijing Genome Institute Europe (Kopenhagen, DK) for providing the sequencing services. We thank the technical personnel of the neurology, neuropathology and radiology departments at Radboudumc and the RWTH University Hospital Aachen for their technical support.

\section{References}

[1] Vorgerd M, van der Ven PF, Bruchertseifer V, et al. A mutation in the dimerization domain of filamin c causes a novel type of autosomal dominant myofibrillar myopathy. Am J Hum Genet 2005;77(2):297-304.

[2] Guergueltcheva V, Peeters K, Baets J, et al. Distal myopathy with upper limb predominance caused by filamin C haploinsufficiency. Neurology 2011;77(24):2105-14.

[3] Duff RM, Tay V, Hackman P, et al. Mutations in the N-terminal actin-binding domain of filamin $\mathrm{C}$ cause a distal myopathy. Am J Hum Genet 2011;88(6):729-40.

[4] Furst DO, Goldfarb LG, Kley RA, et al. Related myopathies: pathology and mechanisms. Acta Neuropathol 2013;125(1):33-46.

[5] Olive M, Kley RA, Goldfarb LG. Myofibrillar myopathies: new developments. Curr Opin Neurol 2013;26(5):527-35.

[6] Kley RA, Hellenbroich Y, van der Ven PF, et al. Clinical and morphological phenotype of the filamin myopathy: a study of 31 German patients. Brain 2007;130(Pt 12):3250-64.

[7] Kley RA, Serdaroglu-Oflazer P, Leber Y, et al. Pathophysiology of protein aggregation and extended phenotyping in filaminopathy. Brain 2012;135(Pt 9):2642-60.

[8] Fischer D, Kley RA, Strach K, et al. Distinct muscle imaging patterns in myofibrillar myopathies. Neurology 2008;71(10):758-65.

[9] Wattjes MP, Kley RA, Fischer D. Neuromuscular imaging in inherited muscle diseases. Eur Radiol 2010;20(10):2447-60. 